\title{
Perceived Social Support and Its Impact on Mental Fatigue in Patients with Mild Traumatic Brain Injury
}

\author{
En quan Zeng ${ }^{1}$, Ben qiang Zeng ${ }^{2}$, Jing lun Tian², Bing Du ${ }^{1}$, Xiao bing Tian ${ }^{1}$, Hong Chen $^{3}$ \\ ${ }^{1}$ North Shichuan Medical College, Nanchong, Sichuan, China \\ ${ }^{2}$ People's Hospital of Wenjiang, Chengdu, China \\ ${ }^{3}$ Affiliated Hospital of North Sichuan Medical College, Nanchong, Sichuan, China
}

\begin{abstract}
Background: Although mental fatigue was well-recognized as one of the long-term consequences following mild traumatic brain injury (MTBI) that required ongoing support, evidences for the optimal management remained inadequate.

Aims: To investigate the temporal profile of mental fatigue during the first year after MTBI and examine the impact of perceived social support on the recovery from post-MTBI fatigue.

Study Design: Observational case-control study.

Methods: This study was conducted among post-MTBI patients admitted to the emergency department in a tertiary-care hospital in Sichuan, China. During four waves of assessments at 1 week, 3, 6 and 12 months, mental fatigue was assessed through Mental Fatigue Scale (MFS) whereas social support was assessed by the Multidimensional Scale of Perceived Social Support (MSPSS).
\end{abstract}

Results: There were $65.1 \%, 37.1 \%, 34.8 \%$ and $32.5 \%$ individuals being identified as those with mental fatigue at 1 week, 3, 6 and 12 months, respectively. The scores of MFS didn't change substantially since 3 months post-injury. Compared to non-fatigued MTBI patients, those with long-lasting post- MTBI fatigue reported extremely lower level of perceived social support. Moreover, improved social support at 1 week was negatively associated with the occurrence of long-lasting fatigue. Conclusion: Sufficient social support could significantly decrease the occurrence of long-lasting mental fatigue among MTBI cases. It seemed of great importance to modify the emphasis of rehabilitation to include assessment and improvement of perceived social support at earlier stages after injury.

Keywords: Mental fatigue, social support, traumatic brain injury
Mental fatigue is one of the most commonly reported symptoms following mild traumatic brain injury (MTBI), with an estimated incidence of $16 \%$ to $73 \%$ across the range of severity of the injury (1-3). It is characterized by rapid mental exhaustion following mental activity and a disproportionally long recovery time after being mentally exhausted $(4,5)$, which may interfere with participation in work, home and social activities and, thereby, reducing quality of life (6). Although several theoretical explanations have been suggested for the cause of mental fatigue (7), the underlying mechanism remains uncertain.
Most persons experiencing post-MTBI fatigue can spontaneously recover shortly after injury (8). However, it may persist for months even years requiring long-term medical care (8). Determining the protocol for the management of longlasting fatigue remains complex and challenging for both clinicians and researchers, as further improvement from this ailment seems very difficult (9). In a randomized controlled trial, early individual intervention by a qualified rehabilitation team didn't appear to improve the outcome of one year after injury among persons with MTBI-related fatigue (10), nor after 10 years (11). Moreover, some studies indicated that intervention 
focusing on post-concussion symptoms (PCS) might in fact increase the awareness of PCS-like symptoms among subjects exposed to such injuries (12). Currently, there remains a dearth of evidence regarding the appropriate management of subjects with long-term post-MTBI fatigue.

It was observed previously that strengthening of social support and improving its perception could alleviate some psychosocial factors including depression and anxiety $(13,14)$, which were identified as common consequences of acquired brain injuries and major contributors of mental fatigue $(8,15)$. Moreover, an intervention on supportive relationships delivered through several rehabilitation programs appeared to result in considerable improvement of life satisfaction and reduction of adverse psychological outcomes $(16,17)$. Given the similar temporal profiles of these mental sequels and their close associations, some researchers argued that these persistent subjective symptoms are not specific enough to be identified as a unique construct and should be considered part of the posttraumatic stress disorder (PTSD) (18). Hence there is a tendency to validate the same rehabilitation modalities for other psychological problems as for mental fatigue (17). However, it remains uncertain due to the lack of studies involving large series of patients under long-term follow-up observations. Therefore, the aim of the present study is, 1) to describe the temporal profile of mental fatigue during the first year after accident, and 2) to examine the association of perceived social support and the occurrence of post-MTBI mental fatigue.

There is therefore the tendency to validate the same prognostic factors for the latter as for such tumors at other site.

\section{MATERIALS AND METHODS}

\section{Study design and participants}

This prospective study was conducted at a universitybased teaching hospital in Sichuan province, China, between January, 2011 and December, 2013. Patients who survived from traffic injuries and admitted to the emergency departments were consecutively screened for eligibility. Permanent residents of the urban area, who were aged between 18 to 60 years and presented with MTBI were eligible for the present study. The definition of MTBI was based on the diagnostic criteria established by the American Congress of Rehabilitation Medicine (19), which consisted of a Glasgow Coma Scale (GCS) score of 13-15 at presentation and post-injury history of loss of consciousness for $<30$ minutes $(19,20)$. Patients were excluded if there were evidences of cerebro-vascular disease, mental retardation, epilepsy, severe systemic medical illness or history of drug/ alcohol abuse.

\section{Measurements}

\section{Mental Fatigue Scale (MFS)}

Participants' mental fatigue was assessed using MFS $(4,6,21)$ which is a multidimensional questionnaire containing 15 questions, covering fatigue in general, lack of initiative, mental fatigue, mental recovery, concentration difficulties, memory problems, slowness of thinking, sensitivity to stress, increased tendency to become emotional, irritability, sensitivity to light and noise, decreased or increased sleep as well as 24-hour symptom variations. Each item comprised examples of common activities to be related to four response alternatives, which could help the person to respond in a similar way despite the present state of fatigue or emotional state. A rating of 0 referred to normal function, 1 indicated a problem, 2: a pronounced symptom and 3: a maximal symptom. It was also possible to provide an answer which fell in between two scores.

Internal consistency and reliability $(a=0.944)$ of the MFS were satisfactory. Observing a significantly slower information processing among MTBI victims as opposed to the healthy controls, Johansson and Ronnback (22), the developers of MFS, recommended a cutoff score at 10.5 for each item of MFS.

\section{The multidimensional scale of perceived social support (MSPSS)}

The MSPSS $(23,24)$ was used to measure the extent to which the social support was perceived by an individual. Having good internal and test-retest reliability, along with robust factorial validity, this scale was proven to be reliable and valid in diverse samples $(23,24)$. It included 12 items with a 7-point likert-type scale, ranging from 'very strongly disagree' to 'very strongly agree' for each item. A total score was calculated by summing the results for all items. Scores ranged between 12 and 84, where higher scores indicated higher level of perceived social support. In addition, separate subscales could be used by summing the responses from the items in each of the three dimensions: family (Item 3, 4, 8 and 11), friends (Item 6, 7, 9, and 12) and significant others (Item 1, 2, 5, and 10). The possible scores in the subscales ranged between 4 to 28 .

\section{Ethics statement}

The study protocol was reviewed and approved by the Institutional Review Committee of the Ethics Board of our institute. Before participation, written informed consent was obtained from each patient after explaining all details of the study in a language that they understand clearly.

\section{Data collection}

Baseline information regarding demographic characteristic and clinical profile of the patients was collected within 1 week 
after injury by reviewing medical records and interviewing the attending physicians. Each participant was then scheduled for 4 waves of assessment by MFS and MSPSS at an interval of 1 week, 3, 6 and 12 months during follow up. Most of the patients were assessed at the out-patient department of NSMC during scheduled re-examination. Others were interviewed over telephone as per prior appointments.

\section{Statistical analysis}

Statistical analysis was performed using statistical analysis software SPSS, version 18.0 (IBM Inc.; Armonk, NY, USA). Participants who completed all the follow-up assessments $(n=169)$ were included in the final analysis. They were further classified into patients with (MFS score $\geq 10.5$ ) or without (MFS score $<10.5$ ) long-lasting MTBI-related fatigue. Categorical variables were described with frequencies and percentages while continuous ones with mean \pm standard deviation (SD). One-way repeated-measure analysis of variance (ANOVA) was carried out to explore how scores of MFS and MSPSS changed over time. Post hoc analysis with Bonferroni's correction was then used to compare MFS and MSPSS scores between two adjacent time points, as well as MSPSS scores between those with or without long-lasting MTBI related fatigue at each time point. To examine the impact of early perceived social support (scores at 1 week) on the occurrence of long-lasting fatigue (reporting fatigue at 12 months), univariate and multivariate logistic regression were conducted to estimate the crude and adjusted associations in terms of Odds Ratios (OR) and corresponding $95 \%$ confidence intervals $(95 \% \mathrm{CI})$.

\section{RESULTS}

Overall 219 eligible patients were included in the initial cohort. Follow-up data was available for 188(85.8\%), 184 $(84.0 \%), 179(81.7 \%)$ and $169(77.2 \%)$ patients at $1^{\text {st }}$ week, $3^{\text {rd }}, 6^{\text {th }}$ and $12^{\text {th }}$ months of assessment, respectively. The participants and the lost-to-follow-up group (those who were present in one assessment but dropped out from the next) did not differ with respect to age, gender, marital and socioeconomic status. Among 169 participants who completed the one year follow up, $96(56.8 \%)$ were men, with mean age of 34.7 years and median education of 12 years (range: 0-22 years). The participants' baseline demographic was presented in Table 1.

According to the cutoff value of 10.5, $110(65.1 \%), 63$ (37.1\%), $59(34.8 \%)$ and $55(32.5 \%)$ individuals were diagnosed with MTBI-related mental fatigue after 1 week, 3, 6 and 12 months follow up, respectively. Moreover, among those having long-lasting MTBI-related fatigue (55 patients having MTBI-related mental fatigue even 12 months after
TABLE 1. Characteristics of the participating post-MTBI patients

\begin{tabular}{lc}
\hline Variables & Values \\
\hline Age at injury in yrs, mean \pm SD & $34.7 \pm 14.8$ \\
Gender, men, n (\%) & $96(56.8)$ \\
Lower education a, n (\%) & $66(39.1)$ \\
Single at the time of injury, n (\%) & $46(27.2)$ \\
Unemployed pre-injury b, n (\%) & $16(9.5)$ \\
Lower annual family income c, n (\%) & $43(25.4)$ \\
\hline
\end{tabular}

SD: standard deviation

a: education years $\leq 9$, b: excluding students, c: $\leq 9,600 \mathrm{RMB}$

TABLE 2. Score of mental fatigue and perceived social support by time of assessment

\begin{tabular}{lccccc}
\hline Variables & 1 week & 3 months & 6 months & 12 months & $\mathrm{p}$ \\
\hline Mental fatigue scale & $12.7 \pm 7.7$ & $9.1 \pm 6.9^{* *}$ & $8.5 \pm 6.1$ & $8.0 \pm 5.9$ & $<0.01$ \\
Social support & & & & & \\
$\quad$ Family & $19.8 \pm 6.0$ & $16.9 \pm 5.9^{* *}$ & $16.3 \pm 5.3$ & $15.4 \pm 4.2$ & $<0.01$ \\
Friends & $21.4 \pm 6.4$ & $20.1 \pm 5.6^{*}$ & $19.5 \pm 5.1$ & $17.0 \pm 5.5^{*}$ & $<0.01$ \\
Significant others & $20.3 \pm 7.4$ & $18.8 \pm 7.2^{* *}$ & $18.4 \pm 6.7$ & $16.3 \pm 5.9^{*}$ & $<0.01$ \\
\hline
\end{tabular}

$* \mathrm{p}<0.05, \quad * * \mathrm{p}<0.01$

Post hoc analysis with Bonferroni's correction was conducted to compare scores of two adjacent time points by item (i.e., 3 months versus 1 week, 6months versus 3 months, and 12 months versus 6 months)

the injury), there were 53 (96.4) patients reporting fatigue at 6 months, $50(90.1 \%)$ at 3 months and $46(83.6 \%)$ at 1 week. As shown in Table 2, the mean score of mental fatigue displayed a significantly decreasing trend during follow up $(\mathrm{F}=10.8, \mathrm{p}<0.01)$. Post hoc test showed that scores at 3,6 and 12 months were significantly lower than that at 1 week, but no marked difference between the three measurements, implying that there were no substantial changes since 3 months after injury. Similarly, the mean score of MSPSS showed an obvious decrease over time at subscale for family $(\mathrm{F}=11.2$, $\mathrm{p}<0.01)$, friends $(\mathrm{F}=9.7, \mathrm{p}<0.01)$ and significant others $(\mathrm{F}=22.5, \mathrm{p}<0.01)$, with the highest score being observed after 1 week and lowest score after 12 months in each subscale. In addition, the mean score for friends and significant others showed significant decrease after 12 months when compared to that of 6 months.

As shown in Table 3, participants with long-lasting fatigue generally reported low levels of perceived social support with reference to those without fatigue. This was reflected in significant differences for the scores in subscales for family $(\mathrm{F}=18.7, \mathrm{p}<0.01)$, friends $(\mathrm{F}=11.8, \mathrm{p}<0.01)$ and significant others $(\mathrm{F}=17.3, \mathrm{p}<0.01)$ between two groups. Although the scores showed a persistent decrease in both groups in these three subscales, the decreasing trend appeared to be more obvious among patients with MTBI-related mental fatigue 
TABLE 3. Comparison of perceived social support between MTBI patients with or without long-lasting fatigue

\begin{tabular}{|c|c|c|c|c|}
\hline Social support & 1 week & 3 months & 6 months & 12 months \\
\hline \multicolumn{5}{|l|}{ Family } \\
\hline Case groupa $(\mathrm{n}=55)$ & $16.5 \pm 4.1$ & $13.5 \pm 4.8^{* *}$ & $12.6 \pm 3.4^{*}$ & $10.3 \pm 3.6^{* *}$ \\
\hline Control groupb $(n=114)$ & $21.7 \pm 5.0$ & $19.0 \pm 4.6^{*}$ & $18.5 \pm 4.1$ & $18.2 \pm 3.3$ \\
\hline \multicolumn{5}{|l|}{ Friends } \\
\hline Case group $(\mathrm{n}=55)$ & $19.6 \pm 4.6$ & $18.5 \pm 4.2^{*}$ & $17.3 \pm 4.2^{*}$ & $14.5 \pm 3.3^{* *}$ \\
\hline Control group $(\mathrm{n}=114)$ & $22.5 \pm 5.4$ & $21.1 \pm 4.8^{*}$ & $20.8 \pm 4.5$ & $19.0 \pm 4.7^{*}$ \\
\hline \multicolumn{5}{|l|}{ Significant others } \\
\hline Case group $(\mathrm{n}=55)$ & $18.0 \pm 5.2$ & $16.8 \pm 4.9^{*}$ & $15.8 \pm 4.4^{*}$ & $12.6 \pm 3.5^{* *}$ \\
\hline Control group $(\mathrm{n}=114)$ & $21.6 \pm 5.5$ & $20.1 \pm 4.3^{* *}$ & $19.7 \pm 4.8$ & $18.4 \pm 4.1$ \\
\hline \multicolumn{5}{|c|}{$\begin{array}{l}{ }^{*} \mathrm{p}<0.05 ; * * \mathrm{p}<0.01 \\
\text { a: participants reporting fatigue at } 12 \text { months; b: participants reporting free of fatigue at } 12 \\
\text { months } \\
\text { Post hoc analysis with Bonferroni's correction was conducted to compare scores of two } \\
\text { adjacent time points (i.e., } 3 \text { months versus } 1 \text { week, } 6 \text { months versus } 3 \text { months, and } 12 \text { months } \\
\text { versus } 6 \text { months) by groups. }\end{array}$} \\
\hline
\end{tabular}

TABLE 4. Association between perceived social support and post-MTBI mental fatigue as determined by logistic regression analyses (simple and multiple)

\begin{tabular}{lcc}
\hline Subscale & cOR $(95 \% \mathrm{CI})$ & aORa $(95 \% \mathrm{CI})$ \\
\hline Family & $0.52(0.36,0.68)$ & $0.64(0.53,0.78)$ \\
Friends & $0.72(0.58,0.92)$ & $0.82(0.68,0.96)$ \\
Significant others & $0.61(0.47,0.75)$ & $0.69(0.56,0.82)$ \\
\hline
\end{tabular}

cOR: crude Odds Ratio; aOR: adjusted Odds Ratio; a: adjusted for age, gender and employment status; CI: confidence interval

compared to those without fatigue, resulting in a gradually increasing gap in scores between these two groups. Post hoc test indicated that the score in non-fatigued group showed obvious change in the 3 months post-injury follow up when compared to that at $1^{\text {st }}$ week in all the three subscales, but no obvious difference was observed since then. However, it persistently decreased in the fatigued group during the whole follow-up period.

Results of simple and multiple logistic regression analyses measuring associations between scores of social support and fatigue development were presented in Table 4. In the unadjusted model, high levels of social support was found to be negatively associated with the occurrence of fatigue in subscale of family (OR $=0.52,95 \%$ CI: $0.36,0.68)$ and friends $(\mathrm{OR}=0.72,95 \% \mathrm{CI}: 0.58,0.92)$. After adjustment for age, gender and employment status, the measures of association (adjusted $\mathrm{OR}=\mathrm{aOR}$ ) became less negative in each scale (for family: aOR $=0.64,95 \%$ CI: $0.53,0.78$; for friends: $\mathrm{aOR}=0.82$, $95 \%$ CI: $0.68,0.96$ and for significant others: $\mathrm{OR}=0.69,95 \%$ CI: $0.56,0.82$ ) but all these associations were found to be statistically significant at $\alpha=0.05$.

\section{DISCUSSION}

Although mental fatigue was well-recognized as one of the long-term consequences following MTBI that required ongoing support, evidences for the optimal management remained inadequate. In this prospective study, we investigated the perceived social support and its relationship with mental fatigue in a sample of individuals with MTBI as a result of traffic accident. Our data revealed that participants with long-lasting fatigue reported obviously low level of social support from family, friends as well as significant others as compared to those without fatigue. Moreover, high level of social support at the early stage appeared to minimize the likelihood of the occurrence of persistent fatigue in these patients. Findings from the present study thus probably could generate valuable insights for the management of MTBI patients to prevent persistent post-MTBI fatigue.

The reported frequency of post-MTBI fatigue varied widely in the background literature, depending on the characteristics of the studied population and the method used to identify fatigue (21). In the present study, to assess MTBI-related fatigue, we employed MFS, a self-report-based scale specific for mental fatigue. The results showed that there were $65.1 \%$, $37.1 \%, 34.8 \%$ and $32.5 \%$ of MTBI patients complaining of fatigue respectively at 1 week, 3,6 and 12 months after injury. These percentages were within the range reported by previous studies and seemed to indicate a higher proportion of occurrence of fatigue among MTBI patients, compared to prior observations (25). Although it seemed not reasonable to compare findings directly between studies, due to the differences in the study objectives and the methodological heterogeneity across studies, these findings consistently indicated that mental fatigue was one of the most important symptoms following MTBI. From the clinical rehabilitation perspective, assessment of mental fatigue was suggested to be one of the key components in clinical management of MTBI patients.

In the present study, we observed a marked decrease in the perception of having social support among MTBI patients during follow up. Patients suffering from long-lasting post-MTBI fatigue complained of extremely lower social support from family, friends and significant others than their counterparts. Similar results were also reported by Izaute et al. (26), that individuals with MTBI were more likely to have significant decrease in perceived social support. Factors contributing towards this decrease might be multidimensional. As one of the less visible consequences following TBI, post-MTBI fatigue was often not recognized immediately after an incident and always not the focus at the beginning of rehabilitation (27). Moreover, it was common for the patients' friends and family members to consider fatigue as 'laziness' or 'apathy'. In a 
longitudinal prospective study, Norrie et al. (8) revealed that a potentially unsupportive interpretation of fatigue-related behaviors as 'laziness' or 'unwillingness' by family members or friends was reported by up to $30 \%$ of all participants. In addition, due to the continuing demands on time and excessive responsibilities of management by patients with persistent fatigue, caregivers of these patients were at increased risk of poor psychosocial outcomes (28). In a study of severe TBI patients and their caregivers, Douglas and Spellacy (29) reported that $60 \%$ of caregivers displayed significant depressive symptoms. It was thus quite difficult for them to provide adequate support to patients with post-TBI fatigue as expected.

However, the perceived availability and quality of social support, as studies indicated, were important predictors for the outcomes of rehabilitation among MTBI patients. In a population-based follow-up study, Stålnacke (16) reported that high level of social support was negatively associated with the probability of occurrence of post-traumatic stress and depression in TBI patients. Izaute et al. (26) indicated that patients with higher perceived level of social support were more likely to have greater satisfaction during rehabilitation, compared to those with lower level of perceived support. In a systematic review, McCabe et al. (30) suggested that rehabilitation, particularly community-based programs using a supportive relationship intervention, could improve level of independence in terms of self-care among individuals with TBI. Similarly, in the current study, it was observed that higher perceived levels of social support in all the three subscales at 1 week after injury were negatively associated with the occurrence of long-lasting fatigue, demonstrating the significant role of perceived social support in improving long-lasting mental fatigue. Evidences from these studies highlighted the importance to modify the emphasis of rehabilitation to include assessment and improvement of actual social support and its perception among TBI patients at earlier stages during recovery after injury.

There were several limitations in this study. Participants were MTBI victims of traffic accidents and their selection was through hospital-based nonrandom sampling. Although studies reported that severity and type of injury seemed not to be the predictors of mental fatigue (15), the impact of these factors on perceived social support was uncertain. So generalization of the study findings to draw conclusion about all TBI patients is not recommended and any extrapolation of the results beyond the study population should be done with caution. Additionally, in the current study complete follow up data on fatigue, on which the fatigue analysis was based, was available for only $77.2 \%$ of the originally included individuals. This might have resulted in an overestimation of the actual prevalence of fatigue, as the participants who continued with the follow up were more likely to be those having fatigue (8).
Finally, although this study was prospective in design, still alike any other observational study, causal interpretation of the observed association between perceived social support and Post-MTBI mental fatigue was not possible. Additional analyses on appropriate data might have helped us in conducting some more analyses to determine the validity of the direction and strength of associations under study but unfortunately were not feasible owing to the nature of our data. So it was unclear whether the improvement of the perception of social support could directly result in the expected decrease in the risk of development of persistent MTBI-related fatigue or not. The potential explanation behind the observed association might include the probability that sufficient perception of social support among MTBI patients increased their ability to participate in their desired activities, improved their quality of life and the sense of control over fatigue and thus alleviated subjective feeling (perception) of fatigue. However, Longitudinal and experimental studies were needed to elucidate the causal pathways for guiding intervention.

In conclusion, the findings of this study indicated that MTBI victims who perceived poor level of social support were at a significantly higher risk of developing post-MTBI fatigue which did not improve significantly since 3 months after injury. On the other hand, sufficient level of perceived social support, during early recovery phase (as early as after 1 week) from MTBI, could significantly decrease the occurrence of longlasting mental fatigue. These findings seemed useful in guiding clinical management of MTBI patients, suggesting the need for rehabilitation programs to include the assessment and improvement of perceived social support for persons with MTBI.

Ethics Committee Approval: Ethics committee approval was received for this study from the North Sichuan Medical College (Protocol No: 2012-14).

Informed Consent: Written informed consent was obtained from patients who participated in this study.

Peer-review: Externally peer-reviewed.

Author contributions: Concept - E.Z., H.C.; Design - H.C.; Supervision - E.Z.; Resource - J.T.; Materials - B.D., B.Z.; Data Collection and/or Processing - X.T., J.T.; Analysis and/or Interpretation - X.T., B.Z.; Literature Search - H.C., E.Z.; Writing - E.Z.; Critical Reviews - H.C.

Acknowledgements: The authors would like to express their sincere gratitude to the medical staff of the emergency departments for their kind assistance in acquisition of data and flied work.

Conflict of Interest: No conflict of interest was declared by the authors. 
Financial Disclosure: The authors declared that this study has received no financial support.

\section{REFERENCES}

1. Sundstrom A, Nilsson LG, Cruts M, Adolfsson R, Van Broeckhoven C, Nyberg L. Fatigue before and after mild traumatic brain injury: pre-post-injury comparisons in relation to Apolipoprotein E. Brain Inj 2007;21:1049-54. [CrossRef]

2. Stulemeijer M, van der Werf S, Bleijenberg G, Biert J, Brauer J, Vos PE. Recovery from mild traumatic brain injury: a focus on fatigue. J Neurol 2006;253:1041-7. [CrossRef]

3. van der Naalt J, van Zomeren AH, Sluiter WJ, Minderhoud JM. One year outcome in mild to moderate head injury: the predictive value of acute injury characteristics related to complaints and return to work. J Neurol Neurosurg Psychiatry 1999,66:207-13. [CrossRef]

4. Johansson B, Berglund P, Ronnback L. Mental fatigue and impaired information processing after mild and moderate traumatic brain injury. Brain Inj 2009,23:1027-40. [CrossRef]

5. Belmont A, Agar N, Azouvi P. Subjective fatigue, mental effort, and attention deficits after severe traumatic brain injury. Neurorehabil Neural Repair 2009;23:939-44. [CrossRef]

6. Marcora SM, Staiano W, Manning V. Mental fatigue impairs physical performance in humans. J Appl Physiol (1985) 2009;106:857-64. [CrossRef]

7. Zgaljardic DJ, Durham WJ, Mossberg KA, Foreman J, Joshipura K, Masel BE, et al. Neuropsychological and physiological correlates of fatigue following traumatic brain injury. Brain Inj 2014;28:389-97. [CrossRef]

8. Norrie J, Heitger M, Leathem J, Anderson T, Jones R, Flett R. Mild traumatic brain injury and fatigue: a prospective longitudinal study. Brain Inj 2010,24:1528-38. [CrossRef]

9. Cantor JB, Ashman T, Bushnik T, Cai X, Farrell-Carnahan L, Gumber S, et al. Systematic review of interventions for fatigue after traumatic brain injury: a NIDRR traumatic brain injury model systems study. J Head Trauma Rehabil 2014,29:490-7. [CrossRef]

10. Elgmark Andersson E, Emanuelson I, Bjorklund R, Stalhammar DA. Mild traumatic brain injuries: the impact of early intervention on late sequelae. A randomized controlled trial. Acta Neurochir (Wien) 2007;149:151-9; discussion 160. [CrossRef]

11. Andersson EE, Bedics BK, Falkmer T. Mild traumatic brain injuries: a 10-year follow-up. J Rehabil Med 2011,43:323-9. [CrossRef]

12. Lange RT, Iverson GL, Brooks BL, Rennison VL. Influence of poor effort on self-reported symptoms and neurocognitive test performance following mild traumatic brain injury. J Clin Exp Neuropsychol 2010;32:961-72. [CrossRef]

13. Matsuda T, Tsuda A, Kim E, Deng K. Association between Perceived Social Support and Subjective Well-Being among Japanese, Chinese, and Korean College Students. Psychology $2014 ; 2014$.
14. Chou KL. Assessing Chinese adolescents' social support: the multidimensional scale of perceived social support. Personality and Individual Differences 2000;28:299-307. [CrossRef]

15. Schnieders J, Willemsen D, de Boer H. Factors contributing to chronic fatigue after traumatic brain injury. J Head Trauma Rehabil 2012;27:404-12. [CrossRef]

16. Stalnacke BM. Community integration, social support and life satisfaction in relation to symptoms 3 years after mild traumatic brain injury. Brain Inj 2007;21:933-42. [CrossRef]

17. Williams MW, Rapport LJ, Millis SR, Hanks RA. Psychosocial outcomes after traumatic brain injury: life satisfaction, community integration, and distress. Rehabil Psychol 2014;59:298-305. [CrossRef]

18. Lagarde E, Salmi LR, Holm LW, Contrand B, Masson F, RibereauGayon R, et al. Association of symptoms following mild traumatic brain injury with posttraumatic stress disorder vs. postconcussion syndrome. JAMA Psychiatry 2014;71:1032-40. [CrossRef]

19. Zasler ND. Mild Traumatic Brain Injury: Medical assessment and intervention. J Head Trauma Rehabil 1993,8:13-29. [CrossRef]

20. Gordon S, Johnson J. ACRM Brain Injury Definition: The Definition of Mild Brain Injury. Available at: http://tbilaw.com/ acrm-brain-injury-definition.html.

21. Schillinger A, Becker F. (Fatigue in patients following traumatic brain injury and stroke). Tidsskr Nor Laegeforen 2015;135:331-5. [CrossRef]

22. Johansson B, Ronnback L. Novel computer tests for identification of mental fatigue after traumatic brain injury. NeuroRehabilitation 2015;36:195-202.

23. Zimet GD, Dahlem NW, Zimet SG, Farley GK. The multidimensional scale of perceived social support. J Pers Assess 1988,52:30-41. [CrossRef]

24. Zhou K, Li H, Wei X, Yin J, Liang P, Zhang H, et al. Reliability and validity of the multidimensional scale of perceived social support in Chinese mainland patients with methadone maintenance treatment. Compr Psychiatry 2015;60:182-8. [CrossRef]

25. Mollayeva T, Kendzerska T, Mollayeva S, Shapiro CM, Colantonio A, Cassidy JD. A systematic review of fatigue in patients with traumatic brain injury: the course, predictors and consequences. Neurosci Biobehav Rev 2014;47:684-716. [CrossRef]

26. Izaute M, Durozard C, Aldigier E, Teissedre F, Perreve A, Gerbaud L. Perceived social support and locus of control after a traumatic brain injury (TBI). Brain Inj 2008;22:758-64. [CrossRef]

27. Cook GA, Hawley JS. A review of mild traumatic brain injury diagnostics: current perspectives, limitations, and emerging technology. Mil Med 2014;179:1083-9. [CrossRef]

28. Ennis N, Rosenbloom BN, Canzian S, Topolovec-Vranic J. Depression and anxiety in parent versus spouse caregivers of adult patients with traumatic brain injury: a systematic review. Neuropsychol Rehabil 2013; 23:1-18. [CrossRef]

29. Douglas JM, Spellacy FJ. Correlates of depression in adults with severe traumatic brain injury and their carers. Brain Inj 2000;14:71-88. [CrossRef]

30. McCabe P, Lippert C, Weiser M, Hilditch M, Hartridge C, Villamere J; Erabi Group. Community reintegration following acquired brain injury. Brain Inj 2007;21:231-57. [CrossRef] 